\title{
Matched Filter Implementation, Complexity, and Gain in Fractional Fourier Domain
}

\author{
S.A. Elgamel ${ }^{*}$ \\ Military Technical College, Cairo, Egypt
}

The manuscript was received on 13 June 2021 and was accepted
after revision for publication as research paper on 30 December 2021.

\begin{abstract}
:
The radar matched filter is implemented in fractional Fourier domain (FrFD) and the required processing steps to perform the radar matched filter in FrFD are demonstrated. The complexity of the FrFD matched filter over the normal frequency transform matched filter is also investigated. The performance enhancements for using the matched filter in the FrFD are presented and the enhancement in the signal to noise ratio (SNR) output at different target SNRs are also described.
\end{abstract}

\section{Keywords:}

chirp radar, fractional Fourier transform, matched filter

\section{Introduction}

Radar matched filters (MF) in time domain (TD) are achieved by correlating the radarreceived signal with a time-reversed radar-transmitted signal. The correlation process in the frequency domain (FD) is executed by multiplying both signals (the received signal and replica signal) before transforming them back into the TD.

In [1], a matched filters in the FT domain and the FrFT domain were investigated using the principle of stationary phase (PSP). The limitation of using matched filter in the optimum FrFT for a chirp was shown to be the dependence of the chirp shape in this domain on the chirp start time and chirp band width. In the special case where specific parameters can be estimated then a significant enhancement results when using matched filter in the optimum FrFT domain compared to the conventional FT approach.

In [2], a generalized fractional matched filtering (GFMF) for estimating higher order chirp parameters with known time delay is presented. A novel method to minimize signal to noise ratio (SNR) gain degradation due to the range-Doppler coupling effect of quadratic chirps is presented. Thus, a joint estimation of time delay and high-

\footnotetext{
* Corresponding author: Electronic Warfare Department, Military Technical College, KobriElkobba, EG-11766 Cairo, Egypt. Phone: +2-22621908, E-mail: elgamel@mtc.edu.eg. ORCID 0000-0003-0627-5729.
} 
er order chirp parameters using generalized fractional envelope correlator (GFEC) are introduced. In [3], the concept of the matched filtering is generalized to the fractional Fourier case. The response of the matched filter in FrFD to chirp signals is analysed, and the sidelobe suppression method for matched filtering in the FrFD is considered.

This paper investigates three methods, used to estimate the time delay $T_{\mathrm{st}}$ in order, to design the MF in the FrFD. The complexity of the FrFD matched filter over the normal Fourier transform (FT) matched filter is also investigated. The performance enhancements in using the matched filter in the FrFD are presented and the enhancement in the output SNRs at different target SNRs are also described.

The paper is organized as follows: the mathematical formula for linear chirp radar received signal in the fractional domain is reviewed in section 2 . In section 3 , the linear chirp start time estimation is discussed. Implementation of the radar MF in FrFD is described in section 4. Discussion underlining the results and the complexity comparison for matched filter in both the FD and FrFD are demonstrated in section 5. A simulation result is presented in section 6 for FrFT and FT matched filter enhancement at different SNRs. Section 7 concludes the paper.

\section{Mathematical Model for Matched Filter in FrFD}

The linear radar chirp received signal representation in the FrFD $S_{\theta}\left(t_{a}\right)$ may be written as [1]:

$$
\begin{aligned}
S_{\theta}\left(t_{a}\right) & =B \operatorname{rect}\left[\frac{t_{a}-\tau u_{\mathrm{o}}^{2} \cos \theta}{\Delta f \sin \theta+u_{o}^{2} T \cos \theta}\right] \exp \left(\phi_{1}+\phi_{2}+\phi_{3}\right) \\
\phi_{1} & =\mathrm{j} \pi t_{a}^{2}\left[\frac{D+\cot \theta u_{\mathrm{o}}^{2}}{\left(D \sin \theta+u_{\mathrm{o}}^{2} \cos \theta\right)^{2}}-\frac{2 \csc \theta}{D \sin \theta+u_{\mathrm{o}}^{2} \cos \theta}+\cot \theta u_{\mathrm{o}}^{2}\right] \\
\phi_{2} & =\mathrm{j} \pi t_{a}\left[\frac{2 D^{2} \tau \sin \theta+2 D \tau \cos \theta u_{o}^{2}}{\left(\sin \theta+u_{\mathrm{o}}^{2} \cos \theta\right)^{2}}-\frac{4 D \tau}{D \sin \theta+u_{\mathrm{o}}^{2} \cos \theta}\right] \\
\phi_{3} & =\mathrm{j} \pi\left[\frac{(D \tau \sin \theta)^{2}\left(D+\cot \theta u_{\mathrm{o}}^{2}\right)}{\left(D \sin \theta+u_{o}^{2} \cos \theta\right)^{2}}-\frac{2 D^{2} \tau^{2} \sin \theta}{D \sin \theta+u_{\mathrm{o}}^{2} \cos \theta}+\tau^{2} D\right]
\end{aligned}
$$

where $B$ is the chirp radar received signal amplitude in FrFD, rect[.] is rectangular function, $\Delta f$ is the chirp bandwidth, $T$ is the radar pulse duration, $t_{a}$ is the variable in the $a^{\text {th }}$ FrFD, and $\theta=a \pi / 2$ with $a \in \Re, u_{\mathrm{o}}$ represent the normalized variables for $t_{a}, \tau$ is the shifted start time, and $\mathrm{D}=\Delta f / T$.

$S_{\theta}\left(t_{a}\right)$ in Eq. (1) is characterized by

$$
\operatorname{rect}\left[\frac{t_{a}-\tau u_{\mathrm{o}}^{2} \cos \theta}{\Delta f \sin \theta+u_{\mathrm{o}}^{2} T \cos \theta}\right]
$$

thus the $a^{\text {th }}$ FrFD order depends on $\tau u_{0}^{2} \cos \theta$. Because the MF implementation in the FrFD depends on the linear chirp shape in the same FrFD, thus MF design in FrFD requires knowledge about $T_{\mathrm{st}}$ which is an unknown parameter in radar application and it can be determined from 


$$
T_{\mathrm{st}}=\frac{2 R_{\mathrm{t}}}{c}
$$

where $R_{\mathrm{t}}$ is the target range, $c$ is the microwave propagation speed, approximate value $3 \times 10^{8} \mathrm{~m} \mathrm{~s}^{-1}$.

In the FD transformation of the FT MF, the signal is characterized by $\operatorname{rect}[f / \Delta f]$ which depends only on $\Delta f$ (known information). Implementation of the radar MF in FrFD. The design of a radar matched filter in the optimum FrFD is a more complex problem than the normal FT MF due to the need for estimating $T_{\mathrm{st}}$. In radar applications, knowledge of the time position of the received useful signal is normally unknown. From FrFT properties, it is evident that a delayed signal will appear to have a shift and a modulation in the FrFD, both depending on the unknown delay of the received signal. This means that without the exact knowledge of the delay of the signal it is impossible to design a matched filter approach directly in the FrFD. Techniques for estimating this signal delay for radar-received signals $T_{\text {st }}$ are presented in the following section.

\section{Methods to Estimate chirp start time}

Estimating the linear chirp start time $T_{\mathrm{st}}$, which is the time of radar received linear chirp, depends on the target range. This estimation is essential in order to design the radar MF in the FrFD. Fig. 1 shows a simulated noisy target linear chirp received signal. The time delay $T_{\text {st }}$ needs to be estimated in order to design the MF in the FrFD through using one of the following three methods:

- FT MF,

- Optimum FrFT of the received linear chirp,

- Optimum FrFT of both the received and a reference signal.

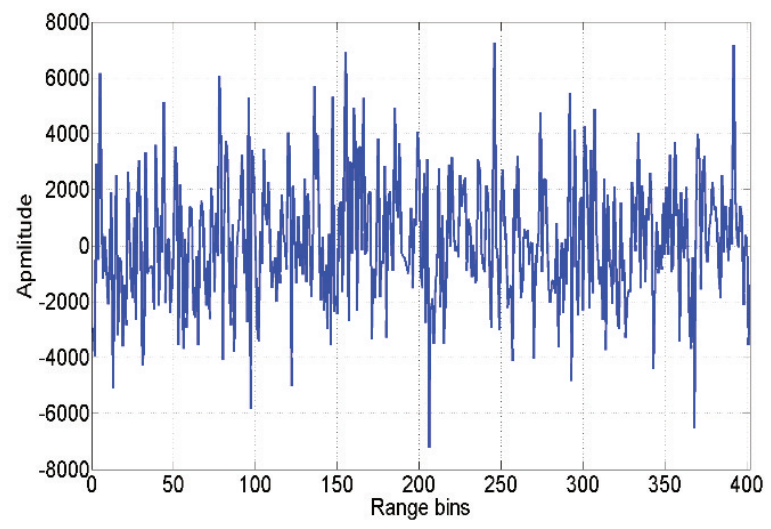

Fig. 1 Noisy linear chirp received signal

\section{Method 1: Fourier Transform Matched Filter}

Applying the MF formula in [1] to the noisy linear chirp radar received signal in Fig. 1, the signal output of the MF is seen in Fig. 2. In Fig. 2, a spike appears at the range bin 150 (related to the target range) which is the start bin of the bins occupied by the received linear chirp. This start bin $R_{\text {bin }}\left(R_{\text {bin }}=150\right)$ is used to calculate the target range $R_{\mathrm{t}}$ which is used to calculate $T_{\text {st }}$ from Eq. (1). 
The target range $R_{\mathrm{t}}$ is calculated from the range bin by adding the range at bin 0 , $R_{0}$, to the distance $\mathrm{d} R$ within the time window as:

$$
R_{\mathrm{t}}=R_{\mathrm{o}}+\mathrm{d} R
$$

and $R_{\mathrm{o}}$ is defined by

$$
R_{\mathrm{o}}=0.5 c T_{w_{1}}
$$

where $T_{w 1}$ is the start of time window and $\mathrm{d} R$ is calculated from:

$$
\mathrm{d} R=\frac{1}{2} c \frac{R_{\mathrm{bin}}-0.5}{F_{\mathrm{s}}}
$$

The calculated target range $R_{\mathrm{t}}$ from Eqs (3)-(5) is $156.975 \mathrm{~km}$, thus the estimated $T_{\text {st }}$ from Eq. (2) is $0.001 \mathrm{~s}$.

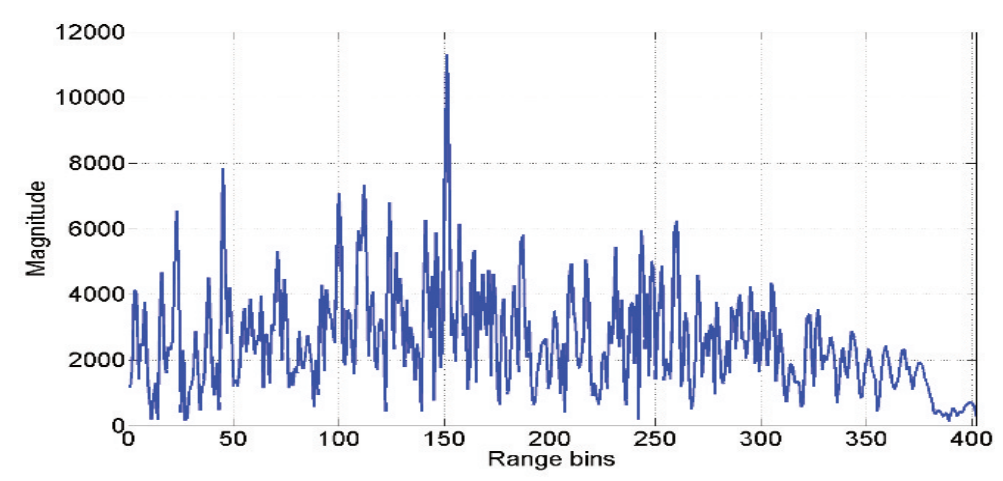

Fig. 2 Estimate $T_{\text {st }}$ using $M F$

\section{Method 2: Optimum FrFT of the Received Linear Chirp}

Fig. 3 shows the radar received signal transformed to the optimum FrFD. A spike at sample 195 is seen in zoomed area of Fig. 3. The linear chirp $P_{p}$ sample number (195) is used to calculate $t_{\mathrm{st}}$ (the linear chirp start time sampling number) from Eq. (6) to be 150 [4].

$$
P_{p}=\sin \vartheta_{\mathrm{opt}}\left[\frac{F_{\mathrm{start}}}{\delta f}+\frac{\Delta f\left(L / M_{T}\right)}{2 \delta f}\right]-\cos \vartheta_{\mathrm{opt}} t_{\mathrm{st}}
$$

where $\vartheta_{\text {opt }}$ is optimum fractional angle $\vartheta_{\text {opt }}=a \pi / 2, F_{\text {start }}$ is the chirp signal starting frequency, $\delta f$ is the frequency resolution $\delta f=F_{\mathrm{s}} / N$, and $N$ is the number of signal samples. $M_{\mathrm{T}}$ is the number of samples in the chirp signal with pulse width $T$.

The sample number $t_{\mathrm{st}}$ is multiplied by the sampling time $T_{\mathrm{s}}=1 / F_{\mathrm{s}}$ to calculate $T_{\mathrm{st}}$ to be the same value 0.001 as in the previous method.

\section{Method 3: Optimum FrFT of Both the Received and a Reference Signal}

In Fig. 4, the radar received signal is transformed to the optimum FrFD as in the previous section, the target spike $P_{p T}$ in the optimum FrFD is observed to be located at 195. A linear chirp signal with $T_{\mathrm{st}}=0$ is used as reference signal that is also transformed to the optimum FrFD, the location of the reference signal spike $P_{p R}$ in the optimum FrFD is at sample 323. $T_{\text {st }}$ is estimated from: 


$$
T_{\mathrm{st}}=\operatorname{abs}\left[\frac{P_{p T}-P_{p R}}{\cos \alpha_{\mathrm{opt}}}\right] / F_{s}+T_{w_{\mathrm{l}}}
$$

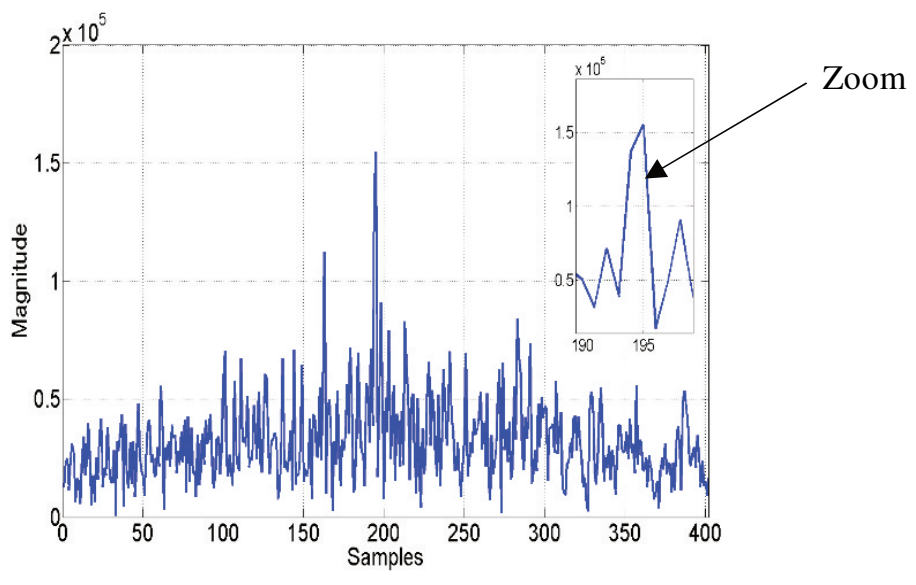

Fig. 3 Estimate $T_{\text {st }}$ using $P_{p}$

For the stating time window $T_{w 1}$ is $8.97 \mu \mathrm{s}$, and calculated optimum angle $\alpha_{\mathrm{opt}}$ is $30.83^{\circ}$ in Eq. (6) the estimated $T_{\mathrm{st}}$ is $0.001 \mathrm{~s}$.

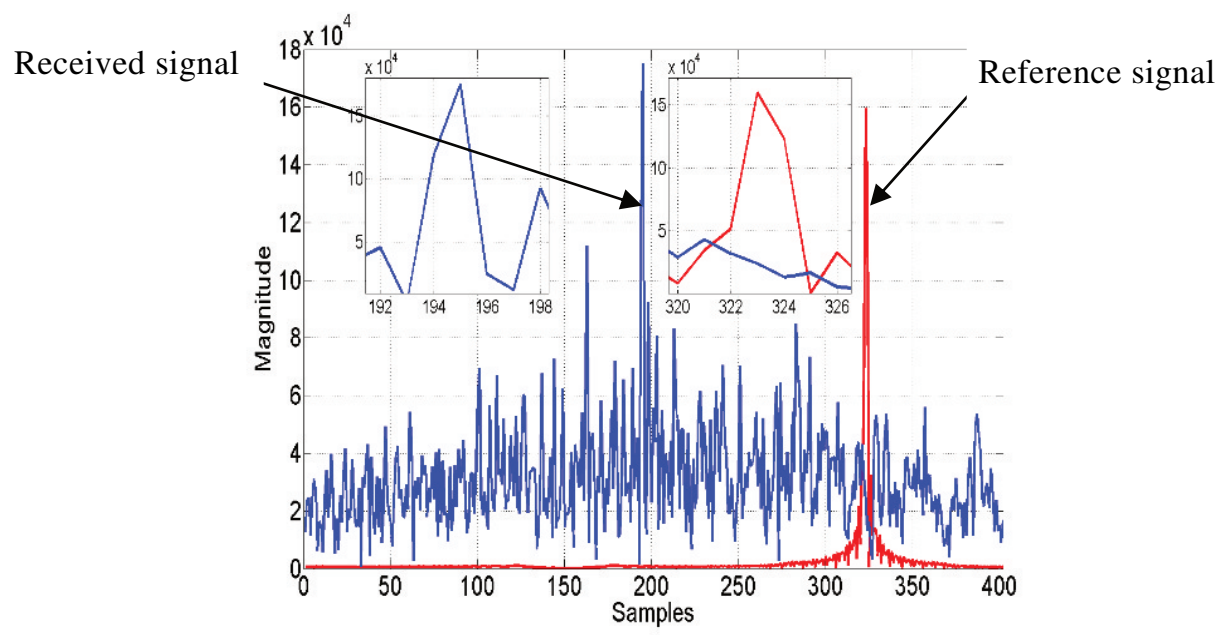

Fig. 4 Estimate $T_{\mathrm{st}}$ using the difference in fractional bins

\section{Implementation of the Radar MF in FrFD}

The radar MF in FrFD is implemented as follows: The received signal, $s(t)$ is transformed to the optimal FrFD using apriori information from the transmitted linear chirp signal. This signal is convolved in the optimum FrFD with a replica signal. The mathematical formula of this replica signal in the time depends on the known or estimated value $T_{\text {st. }}$ The convolution process is achieved by applying FT for both signals in the optimum FrFD, multiplying the two, then applying the inverse Fourier transform (IFT). 
The required processing steps to perform the Radar MF in FrFD are described below:

- optimum transformation order estimation: starting from the transmitting signal parameters (known signal parameters), the optimum fractional order is estimated to transform the received signal to the optimum FrFD,

- FrFT of the received signal: the optimum FrFT of the received signal is performed to obtain a narrow peak detecting the presence of the received signal,

- $T_{\mathrm{st}}$ estimation: the linear chirp start time $T_{\mathrm{st}}$ is estimated by one of the previously described methods. This information is required in order to generate a suitable replica,

- replica generation in the FrFD: using the known or estimated value of $T_{\text {st }}$, a replica of the signal can be generated and its FrFT can be performed,

- FT and matched filtering: to reduce the computational complexity of the matched filtering for the received signal and the replica optimum fractional version, both are transformed into their relative FD before they have been multiplied together,

- inverse Fourier Transform: IFT on the resulting signal after multiplying is performed to produce the matched signal output in the optimum FrFD.

The proposed optimum FrFD radar MF approach can be used for multiple targets detection in the received signal. In the case of multiple targets, the same number of echoes of the transmitted signal as the number of targets illuminated from the system are present in the received signal. A FrFT of the received signal will show the presence of multiple peaks, each one representing a target. In order to apply the fractional $\mathrm{MF}$, an estimation of the unknown time delay must be performed for each target due to the shift variance property, meaning that a different replica must be used to extract each target. The procedure to extract the targets is described as follows:

- to use one of the previously described techniques to estimate the delay of the first echo with significant amplitude,

- to generate the replica with the estimated delay and to apply the fractional MF obtaining the target detection,

- to subtract the first echo from the received signal in order to perform the FrFD MF for the remaining targets. This subtraction can be performed by subtracting the replica used for the FrFD MF from the received signal. The residual received signal will now contain another echo with a significant peak amplitude,

- to iterate the above three steps (1-3) using the residual signal to extract the next target,

- to add the output from the previous iterations to the output of each iteration to obtain the final detections.

The procedure can be iterated a fixed number of times if the number of targets in the received signal is known, or a stop rule for the iterations can be used, i.e. the algorithm can be stopped if the amplitude of the peak in the residual FrFT of the received signal is less than a certain threshold.

\section{Matched Filtering in Fourier Domain versus FrFD}

Performing the Matched filtering in the FrFD is advantageous over the regular FD in that it achieves a narrower main lobe, reduces the side lobe levels, and enhances the 
SNR of the output. The linear chirp parameters $\left(\Delta f, T, F_{\text {start }}\right.$ and $F_{\text {stop }}$, the linear chirp received time in the radar receiving window $T_{\mathrm{st}}$, and the sampling Frequency $F_{\mathrm{s}}$ are the parameters that affect the extent of these enhancements. These improvements when using the proposed MF in the FrFD compared to the MF in the FD face an increased complexity requirement. To compare the Matched filtering complexity in both the Fourier and the FrFDs, it is required to know the complexity of fast Fourier transform (FFT) algorithm and FrFT algorithm that will be described in the following:

- the complexity (complex multiplications) for an $N$-point FFT algorithm is $(N / 2) \log _{2} N$ (for $N$ power of two),

- various methods [5-12] are used to implement the FrFT algorithm. The complexity of these algorithms is approximated to $N \log _{2} N$.

Tab. 1 shows a comparison of complexity between the MF implementation in both FD and FrFD. It shows that the MF in FD implemented by the FFT of the received radar signal multiplied by stored FFT replica of the received signal followed by inverse fast Fourier transform (IFFT) has a complexity approximated by $\mathrm{Nlog}_{2} N$ (the same complexity as the FrFT). The implementation of the MF in FrFD starts with FrFT of the received radar signal multiplied by FrFT replica of the received signal (the mathematical formula of this replica depends on estimation of the start time $T_{\text {st }}$ ) followed by inverse fast Fourier transform. Depending on this implementation, the total complexity for an MF in FrFD as seen in Tab. 1 is approximated by $2.5 N \log _{2} N$. Thus the complexity for implementing the MF in FrFD is approximately 2.5 times the complexity of the MF in Fourier domain.

Tab. 1 Matched filter complexity in the FD and FrFD

\begin{tabular}{|c|c|c|}
\hline Filter & MF based FT & MF based FrFT \\
\hline $\begin{array}{c}\text { Implement } \\
\text { by }\end{array}$ & $\begin{array}{c}1 \text { FFT } \\
1 \text { multiplication } \\
1 \text { IFFT }\end{array}$ & $\begin{array}{c}2 \text { FrFT } \\
1 \text { multiplication } \\
1 \text { IFFT }\end{array}$ \\
\hline \multirow{3}{*}{ Complexity } & $1 N$ (multiplications $)$ & $1 N($ multiplications $)$ \\
& $+0.5 N \log _{2} N(1 \mathrm{FFT})$ & $+2 N \log _{2} N(2$ FrFT $)$ \\
& $+0.5 N \log _{2} N(1 \mathrm{IFFT})$ & $+0.5 N \log _{2} N(1 \mathrm{IFFT})$ \\
\cline { 2 - 3 } & $\approx N \log _{2} N$ & $\approx 2.5 N \log _{2} N$ \\
\hline
\end{tabular}

\section{Matched Filter Outputs at Different SNRs}

Simulation results for a linear chirp signal $\left(\Delta f=30 \mathrm{~Hz}, T_{\mathrm{st}}=2 \mathrm{~s}\right.$ with linear chirp duration $2.5 \mathrm{~s}, \mathrm{~T}=5 \mathrm{~s}$, and $F_{\mathrm{s}}=100 \mathrm{~Hz}$ ) in a noisy environment with different SNRs applied to both FT and FrFT MFs are shown in Fig. 5. This shows improvements in SNR output for the linear chirp signal for the two MFs. The results in Fig. 5 show an average improvement of approximately $3 \mathrm{~dB}$ by using FrFT MF compared to the FT MF for different input SNRs in the range of [-15:20] dB. This gain in performance is accompanied with an increase in complexity, as analysed in section 5 for the implementation of MF in FrFD, as well as with the extra processing required for estimating the radar-received parameters. For different simulation scenarios (different $T_{\mathrm{st}}, \Delta f, T$, $F_{\text {start }}, F_{\text {stop }}$ and $F_{\mathrm{s}}$ ), an SNR improvement is achieved using FrFT MF compared to using the FFT MF. 


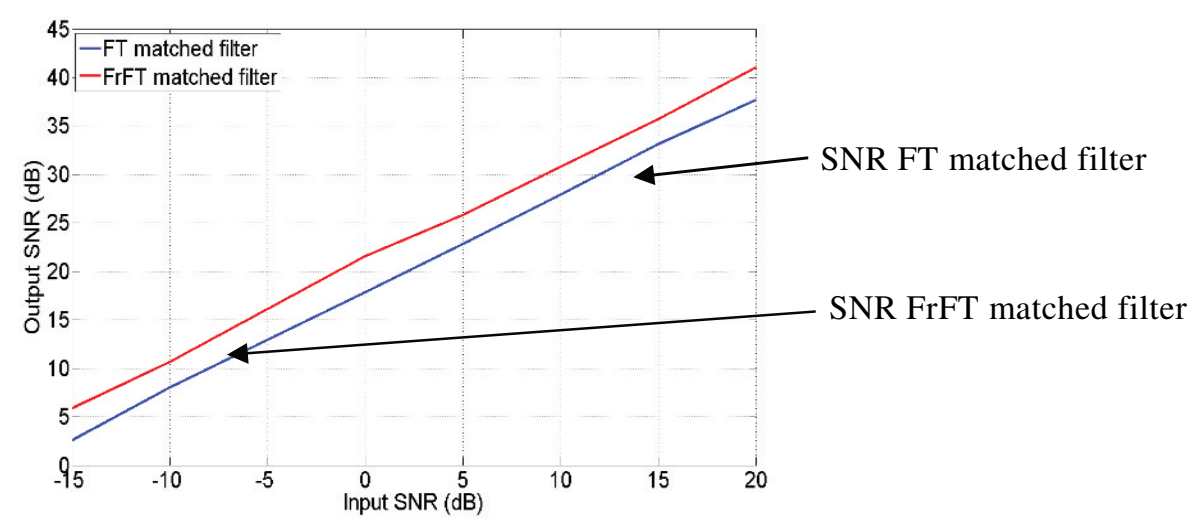

Fig. 5 SNR signals output using FT and FrFT matched filtering

\section{Conclusion}

Mathematical model based on PSP for MF in both the FD and the FrFD were investigated in this paper. It is shown that using MF in the optimum FrFD depends on the linear chirp shape in this domain, on the linear chirp start time and linear chirp bandwidth. The complexity of FrFT MF increases by approximately 2.5 times compared to the normal FT MF. An average improvement of approximately $3 \mathrm{~dB}$ is gained by using FrFT MF compared to the FT MF for different input SNRs in range [-15:20] dB in the considered case. Thus, matched filtering based FrFT can be used for target detection in linear chirp radars with the advantages of increasing the output SNR, reducing the side lobe power, which is applicable for multiple targets detection with the limitation of estimating the target received time $\left(T_{\mathrm{st}}\right)$ and increasing complexity compared to FT based matched filtering.

\section{References}

[1] ElgAMEL, S.A., C. CLEMENTE and J.J. SORAGHAN. Radar Matched Filtering Using the Fractional Fourier Transform. In: Proceedings of the Sensor Signal Processing for Defence (SSPD 2010). London: IET, 2010. DOI 10.1049/ic.2010.0242.

[2] SAHAY, P., A. ANJARLEKAR, S.A. JAIN, P. RADHAKRISHNA and V.M. GADRE. Generalized Fractional Matched Filtering and its Applications. In: Proceedings of the National Conference on Communications (NCC). Kharagpur: IEEE, 2020. DOI 10.1109/NCC48643.2020.9055991.

[3] ZHANG, F., R. TAO and Y. WANG. Matched Filtering in Fractional Fourier Domain. In: Proceedings of the Second International Conference on Instrumentation, Measurement, Computer, Communication and Control. Harbin: IEEE, 2012. DOI 10.1109/IMCCC.2012.9.

[4] JACOB, R., T. THOMAS and A. UNNIKRISHNAN. Applications of Fractional Fourier Transform in Sonar Signal Processing. IETE Journal of Research, 2009, 55(1), pp. 16-27. DOI 10.4103/0377-2063.51320. 
[5] KOC, A., H.M. OZAKTAS, C. CANDAN and M. ALPER KUTAY. Digital Computation of Linear Canonical Transforms. IEEE Transactions on Signal Processing Transactions, 2008, 56(6), pp. 2383-2394. DOI 10.1109/TSP. 2007.912890.

[6] BULTHEEL, A. and H. MARTìnEZ SULBARAN. A Shattered Survey of the Fractional Fourier Transform [online]. 2002 [viewed 2021-01-09]. Available from: https://limo.libis.be/primo-explore/fulldisplay?docid=LIRIAS1945897 $\&$ context=L\&vid=Lirias\&search_scope $=$ Lirias\&tab=default_tab\&lang=en_US\&f romSitemap $=1$

[7] OZAKTAS, H.M., O. ARIKAN, M.A. KUTAY and G. BOZDAGT. Digital Computation of the Fractional Fourier Transform. IEEE Transactions on Signal Processing, 1996, 44(9), pp. 2141-2150. DOI 10.1109/78.536672.

[8] ELGAMEL, S.A. and J. SORAGHAN. A New Fractional Fourier Transform Based Monopulse Tracking Radar Processor. In: IEEE International Conference on Acoustics Speech and Signal Processing (ICASSP). Dallas: IEEE, 2010, pp. 2774-2777. DOI 10.1109/ICASSP.2010.5496208.

[9] ELGAMEL, S.A. and J.J. SORAGHAN. Enhanced Monopulse Radar Tracking Using Filtering in Fractional Fourier Domain. In: Proceedings of the IEEE Radar Conference. Arlington: IEEE, 2010, pp. 247-250. DOI 10.1109/RADAR. 2010.5494618.

[10] CAPUS, C. and K. BROWN. Short-Time Fractional Fourier Methods for the Time-Frequency Representation of Chirp Signals. Journal of the Acoustical Society of America, 2003, 113(6), pp. 3253-3263. DOI 10.1121/1.1570434.

[11] LEITH, E. Review of 'Systems and Transforms With Applications to Optics' IEEE Transactions on Information Theory, 1972, 18(3), pp. 451-452. DOI 10.1109/TIT.1972.1054814.

[12] AMEIN, A.S. and J.J. SORAGHAN. Fractional Chirp Scaling Algorithm: Mathematical Model. IEEE Transactions on Signal Processing, 2007, 55(8), pp. 4162-4172. DOI 10.1109/TSP.2007.895994. 